\title{
Renforcement des données probantes et des mesures relatives aux partenariats plurisectoriels en santé publique : un projet de recherche-action
}

\author{
C. D. Willis, Ph. D. (1, 2, 3); J. K. Greene, M. Éd. (2); A. Abramowicz, M. Sc. (1); B. L. Riley, Ph. D. (1)
}

Cet article a fait l'objet d'une évaluation par les pairs.

Diffuser cet article sur Twitter

\section{Résumé}

Introduction : L'initiative Partenariats plurisectoriels de l'Agence de la santé publique du Canada, gérée par le Centre de prévention et de contrôle des maladies chroniques (CPCMC), réunit différents partenaires en vue de concevoir, de mettre en œuvre et de faire progresser des approches novatrices visant à améliorer la santé de la population. Cet article décrit l'évolution et les premières priorités d'un projet de recherche-action (stratégie d'apprentissage et d'amélioration) dont l'objectif est de faciliter l'amélioration continue de l'initiative de partenariat du CPCMC et de contribuer aux données probantes sur les partenariats plurisectoriels.

Méthodologie : La stratégie d'apprentissage et d'amélioration du CPCMC pour les partenariats plurisectoriels repose sur les consultations avec le personnel et la haute direction du CPCMC et sur l'examen de cadres conceptuels liés aux partenariats plurisectoriels. Les consultations ont porté sur l'élaboration de l'initiative plurisectorielle, ses obstacles, ses facteurs de réussite et ses indicateurs d'efficacité. Nous avons fait une revue de littérature et consulté la littérature grise en appliquant une stratégie de recherche systématique puis nous avons résumé nos conclusions sous forme de comptes rendus.

Résultats : Les consultations et l'examen de la littérature ont souligné l'importance de comprendre l'incidence des partenariats, d'élaborer une vision commune, de mettre en œuvre un système partagé de mesures et de créer des occasions d'échange de connaissances. C'est dans cette perspective que nous proposons une stratégie d'apprentissage et d'amélioration en six volets : 1) donner la priorité aux besoins en matière d'apprentissage, 2) relier les besoins aux données probantes, 3) utiliser des méthodes de collecte de données pertinentes, 4) analyser et synthétiser ces données, 5) fournir une rétroaction aux membres et aux équipes du CPCMC et 6) agir. Les premiers besoins en matière d'apprentissage concernent la portée des partenariats et les répercussions non anticipées des partenariats plurisectoriels sur les individus, les équipes, les organismes et les communautés.

Conclusion : Bien que principalement destinée au CPCMC, la stratégie d'apprentissage et d'amélioration pourrait s'avérer tout aussi pertinente pour d'autres publics, notamment d'autres ministères ou organismes externes intéressées à saisir et partager de nouvelles connaissances sur les partenariats plurisectoriels.

Mots-clés : partenariats plurisectoriels, collaboration, amélioration continue,

apprentissage

\section{Points saillants}

- Bien que les partenariats multisectoriels sur les enjeux complexes en santé ne soient pas nouveaux, nous n'en n'avons qu'une connaissance limitée.

- Nous avons créé une stratégie d'apprentissage et d'amélioration pour augmenter les connaissances sur l'initiative des partenariats plurisectoriels de l'Agence de la santé publique du Canada, pour en améliorer les effets et pour explorer des enjeux nouveaux et d'actualité dont on ne rend habituellement pas compte dans les activités de surveillance et d'évaluation.

- Cette stratégie souligne l'importance de comprendre les effets de ces partenariats, de développer une vision commune, d'implanter un système de mesures partagées et de favoriser les échanges de connaissances.

- Cette stratégie aidera la collecte d'une information pertinente et actualisée, au service des efforts de l'ASPC et de sa base de données probantes sur les partenariats plurisectoriels, utilisable par des publics variés.

\section{Introduction}

Pour parvenir à traiter des questions complexes de santé publique, comme la prévention primaire des maladies chroniques, il est nécessaire que les différents secteurs - en particulier les institutions publiques et privées - adoptent des mesures conjointes et coordonnées $^{1-4}$. Ces efforts de collaboration reposent sur le principe qu'aucun organisme

Rattachement des auteurs :

1. Centre pour l'avancement de la santé des populations Propel, Université de Waterloo, Waterloo (Ontario), Canada

2. Centre de prévention et de contrôle des maladies chroniques, Agence de la santé publique du Canada, Ottawa (Ontario), Canada

3. School of Population Health, Université d'Adelaide, Adelaide (Australie méridionale), Australie

Correspondance: Cameron D. Willis, Menzies Centre for Health Policy, D17 Charles Perkins Centre, Université de Sydney NSW 2006, Australie; courriel : cameron.willis@sydney.edu.au 
et aucun secteur n'a à lui seul la responsabilité ou la capacité d'améliorer la santé de la population. Ce n'est que par un travail fondé sur la collaboration que l'on peut tirer le maximum des ressources, des compétences et des talents afin de réaliser des progrès durables dans les domaines de la prévention et du contrôle des maladies chroniques, en particulier les cancers, les cardiopathies et les maladies mentales ${ }^{4-8}$.

C'est dans cette perspective que le Centre de prévention et de contrôle des maladies chroniques (CPCMC) de l'Agence de la santé publique du Canada (ASPC) a lancé, en 2013, l'initiative Partenariats plurisectoriels pour promouvoir les modes de vie sains et prévenir les maladies chroniques (http://www.phac-aspc.gc.ca/fo-fc/mspphlpppmvs-fra.php). Cette initiative appuie de nombreux projets de partenariats conclus entre des organismes publics et privés dans le but de stimuler le recours à des interventions fondées sur des données probantes traitant des facteurs de risque courants des maladies chroniques ${ }^{9}$. Pour maximiser les bénéfices de cette initiative, le CPCMC a mis au point une stratégie d'apprentissage et d'amélioration permettant d'explorer des enjeux nouveaux et d'actualité dont on ne rend habituellement pas compte dans les activités de surveillance et d'évaluation.

Dans cet article, nous décrivons les différents volets de la stratégie d'apprentissage et d'amélioration destinée aux partenariats plurisectoriels, les processus qui ont servi à son élaboration ainsi que les premières priorités en matière d'apprentissage du CPCMC.

\section{Partenariats plurisectoriels pour promouvoir les modes de vie sains et prévenir les maladies chroniques}

L'initiative Partenariats plurisectoriels pour promouvoir les modes de vie sains et prévenir les maladies chroniques, gérée par le CPCMC, regroupe les investissements fédéraux et ceux du secteur privé et des organismes de bienfaisance ou sans but lucratif, afin de diversifier et d'accrồtre les investissements financiers en prévention des maladies chroniques, de partager les risques potentiels et les bénéfices mutuels entre les organismes participants ainsi que d'accroître la portée et l'incidence des interventions en prévention des maladies chroniques. Elle permet aux partenaires de co-créer des projets, de co-investir en eux et, graduellement, de les co-gérer.

Pour mettre en œuvre cette initiative, le CPCMC a transformé certains de ses investissements en subventions et en contributions. Par exemple, il a délaissé l'habituel processus d'appel d'offre à durée limitée - où les soumissionnaires ne sont acceptés que pendant certaines périodes chaque année - pour adopter plutôt un processus continu à deux étapes permettant la poursuite de partenariats et l'élaboration suivie de projets. De plus, on a adopté un modèle de rémunération au rendement afin d'accroître la responsabilisation : les versements ne sont faits que lorsque les jalons conjointement négociés $\mathrm{du}$ projet sont atteints. Les paiements d'étapes sont fondés sur les résultats du projet, par exemple la mise en œuvre d'une intervention dans un nombre convenu de sites, la réalisation des évaluations exigées (comme la soumission de toutes les données de référence) ou l'élaboration de ressources pour le projet (comme un portail Web, une application mobile ou une plateforme pour les formateurs intégrant l'ensemble des objectifs du projet). De plus, en vue d'élaborer une base de données solide pour les projets financés, le CPCMC a mis en place une méthode permettant de recueillir de façon continue des données sur les changements comportementaux, avec la possibilité d'explorer le retour sur investissement des projets du point de vue social.

Depuis son lancement en février 2013, l'initiative a permis la mise en œuvre de 22 projets de partenariat (axés sur l'activité physique, les modes de vie sain, le tabagisme ou la prévention des blessures, ou encore sur plusieurs facteurs de risque) et le versement de plus de 38 millions de dollars.

\section{Avantages et défis des partenariats plurisectoriels}

Bien que les partenariats plurisectoriels constituent une part importante de l'infrastructure en santé publique, au Canada comme ailleurs dans le monde $\mathrm{e}^{10-16}$, il demeure difficile de les définir, d'en gérer les avantages et les inconvénients, d'en évaluer la structure, les processus et les résultats et enfin d'en améliorer le rendement.

Les partenariats sont souvent vus comme des liens dyadiques entre des organismes qui se consacrent à " l'échange de pouvoir, de tâches, d'aide ou d'information avec les autres en vue d'atteindre des objectifs ou des avantages communs " [traduction] $]^{17,}$ p. 61 . Ces liens forment la base d'autres structures collaboratives, notamment de réseaux (« un groupe d'au moins trois organismes liés de sorte à faciliter l'atteinte d'un objectif commun » [traduction] ${ }^{4}$ ), d'alliances (qui sont « habituellement des partenariats dyadiques qui sont moins complexes et de durée plus courte que les réseaux » [traduction] $^{4}$ ) et de coalitions de communautés (à savoir « des communautés précises ainsi que leurs membres qui représentent la diversité et la sagesse de ces communautés, tant à petite qu'à grande échelle » [traduction] ${ }^{18}$. Quelle que soit la structure de collaboration, les organismes qui en font partie affichent des similitudes et des différences à trois égards : les secteurs qu'ils représentent, les ressources qu'ils apportent et leur secteur d'intervention.

En dépit des différences entre ces initiatives de collaboration, les partenariats présentent tous une série de bénéfices et de risques. C'est particulièrement le cas des partenariats auxquels des organismes privés et publics participent, comme le Right to Play Canada, le Partnership for a Healthier America, le Partenariat canadien pour une vie active après l'école et le Let's Move! Active Schools ${ }^{1-3,19-28}$. Parmi les avantages cités, on compte une capacité accrue à partager les risques et les bénéfices, la sensibilisation d'un plus grand nombre de personnes, d'organismes, de secteurs et de communautés et, grâce aux ententes de partenariat qui ont été prises, l'accroissement de la responsabilisation et de l'engagement des organismes participants ${ }^{2}$. Dans le cadre de partenariats impliquant d'importants organismes privés, on est soucieux des motivations des partenaires industriels, de l'éventuelle présence de conflits d'intérêts, de décalages potentiels 
entre les produits de l'entreprise et les besoins de la communauté, du risque de détournement des priorités gouvernementales en faveur des intérêts du secteur privé, de la possibilité de répercussions négatives sur la réputation (surtout pour le secteur public ou sans but lucratif), de l'éventuel déséquilibre de pouvoirs entre les organismes partenaires et la perte d'autonomie (surtout pour les partenaires moins puissants $)^{1,23}$. Il est donc important de préciser, d'élaborer, d'évaluer et de partager les expériences de ceux qui contribuent à l'établissement, à la gestion et à la surveillance de ces partenariats plurisectoriels, afin de mieux comprendre la façon dont ils fonctionnent dans différents contextes et avec divers partenaires, de même que leur incidence à court et à long terme sur les individus et les populations.

\section{Une occasion d'apprentissage et d'amélioration}

$\mathrm{Au}$ nom de l'ASPC et conformément à son exigence de mesures fondées sur des données probantes, le CPCMC investit dans une stratégie d'apprentissage et d'amélioration en vue de mieux comprendre les partenariats plurisectoriels établis en santé publique, pour améliorer et renforcer la base de données sur les partenariats. Cette stratégie sera également profitable à d'autres centres de l'ASPC, de même qu'à d'autres ministères, organismes, sociétés d'État ou organismes de services spéciaux au sein du gouvernement, ou encore à d'autres organismes (organismes de financement de la recherche, universités, fondations, industries privées, etc.) ayant l'intérêt ou la responsabilité de mieux connaître les partenariats plurisectoriels et de les améliorer. Ce travail s'appuie sur des traditions disciplinaires bien ancrées (comme l'apprentissage organisationnel) éclairant les processus qui permettent aux individus et aux institutions de modifier leurs modèles mentaux, leurs normes, leurs stratégies et leurs presupposes ${ }^{29,30}$. Comme l'a souligné Senge $^{29}$, les organismes favorisant l'apprentissage sont ceux « où chacun améliore constamment sa capacité à créer les résultats qu'il souhaite réellement obtenir, où on favorise les façons de penser nouvelles et en développement, où les aspirations collectives sont libres et où les gens apprennent continuellement ensemble à avoir une vue d'ensemble » [traduction] $^{29, \text { p. } 3}$. Utilisant les facteurs favorisant l'apprentissage organisationnel (comme l'apprentissage en équipe, la pensée systémique et l'établissement d'une vision commune), cet article décrit l'élaboration et les éléments clés de la stratégie d'apprentissage et d'amélioration du CPCMC pour les partenariats plurisectoriels, et il donne un aperçu des premières priorités en matière de mise en œuvre $e^{29,30}$.

\section{Méthodologie}

L'élaboration de la stratégie d'apprentissage et d'amélioration repose sur :

- le personnel du CPCMC ayant participé à la conception et à la mise en œuvre de l'initiative sur les partenariats plurisectoriels et

- l'analyse de cadres conceptuels de partenariats plurisectoriels.

\section{Consultations auprès $d u$ personnel $d u$ CPCMC}

Les consultations réalisées auprès du personnel du CPCMC ont été réalisées par CDW et JKG le 21 mars 2014. Un guide de consultation a été élaboré à la lumière des discussions avec les responsables du projet, de l'examen des documents d'orientation du CPCMC (Guide de partenariat, Cadre d'évaluation des partenaires potentiels, Document d'orientation à l'intention des bénéficiaires d'accords de contribution - Mesurer l'incidence) et de l'examen de documents stratégiques récents de l'ASPC. Le guide de consultation contient une série de questions destinées à recueillir les expériences des participants à propos des partenariats plurisectoriels, des objectifs attendus de l'initiative plurisectorielle de l'ASPC, des points forts et des points faibles des approches actuelles de surveillance et d'évaluation des partenariats, des incertitudes qui restent à éliminer à l'aide de la documentation sur les partenariats plurisectoriels, des premières priorités en matière d'apprentissage ainsi que des spécificités qu'ils souhaitent voir à une initiative d'apprentissage et d'amélioration pour les partenariats plurisectoriels du CPCMC.
Dix-sept membres du personnel du CPCMC ont participé aux consultations dans le cadre de quatre groupes de discussion, chacun comptant entre deux et dix participants. Les groupes pertinents devaient déjà posséder des connaissances et de l'expérience dans la mise en œuvre ou l'évaluation de projets de partenariats plurisectoriels. On a donc invité dans ces groupes les membres qui avaient une vue d'ensemble du programme (directeur général et directeur principal), ceux de la Division des partenariats et des stratégies, ceux de la Division de la mesure du rendement et ceux de la Division des interventions et des pratiques exemplaires.

On a regroupé en un fichier les notes prises au cours des quatre groupes de discussion en personne, animés conjointement par CDW et JG. Une analyse thématique a été effectuée afin d'éliminer les points redondants et de dégager les grands thèmes. Les thèmes retenus ont soit été définis par plusieurs groupes au cours des consultations, soit été jugés très importants par la haute direction. Ils ont fait l'objet de discussions puis ont été retravaillés par un groupe de huit membres du CPCMC ayant aussi participé aux groupes de discussion. Ces membres ont été sélectionnés en raison de leurs rôles et responsabilités (p. ex. établissement des partenariats, évaluation, gestion et surveillance des partenariats, contrats), de l'ampleur de leurs connaissances et expériences ainsi que de leur capacité à favoriser le changement.

\section{Analyse des cadres conceptuels pertinents dans le cas de partenariats plurisectoriels}

Se basant sur les conclusions des consultations, le groupe de travail du CPCMC et les chercheurs du Centre pour l'avancement de la santé des populations ont fixé conjointement l'objectif de l'analyse : déterminer et décrire les cadres ou modèles conceptuels pertinents permettant de comprendre et d'expliquer les caractéristiques, les fonctions et les conséquences (prévues ou non) des partenariats plurisectoriels, que ce soient des liens dyadiques entre deux organismes (indépendamment des secteurs) ou des liens plus étendus (ce que l'on qualifie souvent de réseau interorganisationnel). 


\section{Stratégie de recherche}

Nous avons restreint notre stratégie de recherche à la littérature ayant fait l'objet d'une évaluation par les pairs et à la littérature grise publiée en anglais depuis 2000. On a également consulté cinq bases de données électroniques, PubMed, Academic Search Premier, ABI Inform, Scopus et Web of Science. Notre stratégie, adaptée à chaque base de données, a consisté à utiliser une combinaison de vocabulaire contrôlé et de termes libres (tableau 1). Les termes de recherche ont été regroupés en trois grandes catégories ayant à voir avec des cadres ou des modèles, des initiatives plurisectorielles et des partenariats organisationnels. Les recherches ont d'abord été réalisées séparément, puis ont été rassemblées afin de repérer les modèles conceptuels pertinents aux partenariats à représentants plurisectoriels.

Un examinateur a ensuite éliminé les articles jugés non pertinents à la lumière de leurs titres, et deux examinateurs ont évalué les résumés des autres articles, en réglant les divergences d'avis grâce à des discussions ouvertes (voir le tableau 2 pour les critères d'inclusion et d'exclusion). Les articles qui ne décrivaient aucun cadre, modèle conceptuel ou modèle théorique permettant de comprendre les travaux plurisectoriels ou multiorganisationnels, ou qui ne portaient que sur un seul enjeu ou groupe clinique (p. ex. une maladie ou un groupe professionnel précis), ont été exclus.

Nous avons aussi consulté la littérature grise des sites Web du GSA, du Centre de collaboration nationale des méthodes et outils, du Stanford Social Innovation Review et du Tamarack Institute for Community Engagement, à la recherche de rapports et de publications susceptibles de décrire une application possible de cadres à des initiatives plurisectorielles ou à l'élaboration de stratégies d'apprentissage et d'amélioration pour des partenariats plurisectoriels. On a également consulté Internet à l'aide des moteurs de recherche Google (https://www.google.ca/) et DuckDuckGo (https://duckduckgo.com/), en utilisant des termes clés tirés de la base de données. L'équipe a passé en revue les cinq premières pages de chaque résultat de recherche afin de repérer les documents les plus pertinents. Nous avons appliqué aux recherches sur Internet et à l'examen des sites Web les mêmes critères que ceux de l'évaluation par les pairs (tableau 2).

\section{Extraction et synthèse des données}

Ont été extraites de chacun des documents pertinents repérés au cours de la recherche les données suivantes : nom du cadre, but ou perspective du cadre, éléments essentiels ou facteurs de réussite, domaines d'évaluation (incluant les exemples d'indicateurs s'ils étaient fournis). On a regroupé les cadres selon des perspectives macro, méso et micro. Les cadres à l'échelle macro fournissent une orientation de haut niveau pour comprendre l'incidence sociale d'une collaboration plurisectorielle. Les cadres à l'échelle méso décrivent le fonctionnement de la collaboration ainsi que les facteurs importants pour établir des partenariats.

TABLEAU 1

Catégorisation des termes de recherche

\begin{tabular}{lll} 
Catégorie 1 & \multicolumn{1}{c}{ Catégorie 2 } & Catégorie 3 \\
\hline cadre & plurisectoriel & partenariat \\
modèle & plurilatéral & réseau \\
concept & interorganisme & collaboration \\
méthode & interorganisationnel & plateforme \\
théorie & intersectoriel & alliance \\
théories & multisectoriel & système \\
incidence & & \\
efficacité & & \\
résultat & & \\
rendement & & \\
\hline
\end{tabular}

Enfin, les cadres à l'échelle micro précisent les grands domaines nécessaires à la compréhension du fonctionnement des partenariats, de même que des mesures, des outils et des indicateurs spécifiques plus clairs.

Les cadres relevant de plus d'une échelle ont été classés selon le plus haut niveau d'application.

Pour chaque cadre, les données ont été extraites, compilées puis résumées sous forme narrative.

\section{Élaboration de la stratégie d'apprentissage et d'amélioration}

$\mathrm{Au}$ cours des consultations, le personnel du CPCMC a commencé à faire la liste des défis concrets liés à l'établissement, à la mise en œuvre et à l'évaluation de partenariats plurisectoriels, ainsi que des principaux domaines où l'on ne recueille habituellement pas d'information au cours de la surveillance et de l'évaluation habituelles. Le personnel du CPCMC a également souhaité avoir une meilleure compréhension des facteurs majeurs de réussite des partenariats interorganisationnels, des secteurs clés de mesure et des techniques d'évaluation du rendement des partenariats. Compte tenu de ces intérêts, la revue de littérature a porté sur les cadres des partenariats qui synthétisaient les concepts relatifs aux partenariats plurisectoriels. Afin d'intégrer les données concrètes obtenues lors des consultations du CPCMC et l'information conceptuelle tirée de la revue de littérature, nous avons élaboré une stratégie d'ensemble reposant sur les données à propos des partenariats plurisectoriels tout en répondant aux besoins du personnel du CPCMC. La connaissance des cycles d'apprentissage ${ }^{31}$ et de la manière d'améliorer sans cesse la qualité $^{32}$ a facilité la génération d'une stratégie flexible, itérative et adaptée au contexte précis des partenariats plurisectoriels, fondée sur des cycles d'apprentissage de 3 à 4 mois, ce qui assure une bonne capacité de réaction face aux enjeux exigeant une réponse rapide et l'utilisation optimale des ressources disponibles.

L'équipe CPCMC-Propel a élaboré une version provisoire de la stratégie, qui a 
TABLEAU 2

Critères d'inclusion et d'exclusion

Critères d'inclusion

Le document décrit un cadre, un modèle conceptuel ou un modèle théorique permettant de comprendre les travaux plurisectoriels ou multiorganisationnels.

été ensuite retravaillée par discussions avec 33 membres du CPCMC.

\section{Résultats}

\section{Consultations}

Les consultations ont permis de repérer une série de concepts, d'idées et de besoins liés à l'initiative de partenariats plurisectoriels de l'ASPC, notamment en ce qui a trait à la compréhension de l'incidence, de la conception, de l'établissement et de l'amélioration des partenariats. Trois thèmes principaux ont été dégagés :

(1) Certaines des répercussions de l'initiative de partenariats plurisectoriels et de ses projets apparaissent dans les résultats à court et à long terme obtenus grâce au système de mesure de rendement du projet. Les autres relèvent de l'exploitation (ressources, compétences, réputation, crédibilité, fonds, etc.), de la portée du programme, de la durabilité des interventions, de l'appui à l'innovation sociale, du rendement social et de la crédibilité de l'ASPC (interne et externe au gouvernement). Des effets multiples, tant positifs que négatifs, sont susceptibles d'émerger, notamment une meilleure capacité au sein des organisations partenaires ( $p$. ex. en générant de la valeur sociale), l'acquisition de nouvelles compétences par le personnel des organismes partenaires (p. ex. en évaluation), une meilleure utilisation de la technologie (p. ex. amélioration des techniques de saisie et de surveillance des données), une aggravation potentielle des inégalités en matière de santé en fonction des
Critères d'exclusion

L'article ne décrit aucun cadre, modèle conceptuel ou modèle théorique.

Le document décrit un cadre, un modèle conceptuel ou un modèle théorique qui ne permet pas de comprendre les travaux plurisectoriels ou multiorganisationnels.

Le document n'aborde qu'un sujet ou groupe clinique (p. ex. une maladie ou un groupe professionnel précis). compétences et de formation. Compte tenu du mandat de l'ASPC, de l'expérience offerte par le projet plurisectoriel ainsi que de la demande croissante en matière de données à propos de l'engagement intersectoriel envers l'amélioration de la santé de la population, on a considéré comme particulièrement important de réfléchir aux expériences vécues et d'en tirer des leçons en temps réel.

\section{Analyse des cadres}

conditions socioéconomiques, de la culture ou de localisation et enfin un centrage réduit à la santé des personnes plutôt que portant sur les approches axées sur la santé de la population.

(2) Le CPCMC - notamment au sein de la Division des partenariats et des stratégies et du Bureau de direction, parmi les gestionnaires expérimentés - possède un large éventail de connaissances pratiques sur les types de partenaires et de partenariats, sur ce qui fonctionne (ou pas) pour chacun d'eux, pour qui ils fonctionnent, dans quelles circonstances et pour quelles raisons. Ces connaissances touchent à la façon de démarrer, consolider, soutenir, modifier, mesurer, gérer et évaluer les partenariats plurisectoriels à différents stades de leur évolution, ainsi qu'à la façon dont la structure ou la conception organisationnelle du CPCMC et de l'ASPC contribue - ou nuit - à la pratique actuelle. Quoique des outils, des modèles et des méthodes existent pour éclairer le processus décisionnel, les connaissances (pourtant particulièrement pertinentes) de certains spécialistes ne sont pas systématiquement emmagasinées, partagées ou utilisées pour expliquer, comprendre et améliorer les partenariats plurisectoriels.

(3) L'accent ayant été davantage mis sur la mise en œuvre de l'initiative de partenariats plurisectoriels et de ses projets connexes, les occasions ont été rares au sein du CPCMC de réfléchir aux connaissances, atouts et expériences déjà acquis, d'en tirer profit et d'agir à partir d'eux. Il faut notamment définir et combler rapidement les lacunes en matière de connaissances, de
Des 5363 articles repérés au cours de notre stratégie de recherche, 5066 ont été éliminés en raison de leur titre. Il en est donc resté 297, dont nous avons analysé le texte intégral ou le résumé. Cela a conduit à éliminer 204 articles, en en conservant donc 93. Nous avons encore examiné les résultats de cette recherche, afin de repérer les articles les plus pertinents et les plus récents et d'éliminer les doublons, ce qui a permis de retirer 17 articles publiés avant 2007. Nous avons, pour terminer, évalué la pertinence des 76 articles restants et décidé ou non de les inclure pour leur cadre ou son équivalent, ce qui a conduit à l'élimination de 56 autres.

La consultation de la littérature grise a permis de repérer 26 documents, dont 12 ont été éliminés conformément aux mêmes critères que précédemment, ce qui en a laissé 14.

En tout, nous avons analysé 34 articles, sur 19 cadres différents se rapportant à l'élaboration d'une stratégie d'apprentissage et d'amélioration pour les partenariats plurisectoriels. Le tableau 3 présente les 19 cadres aux échelles macro, méso et micro inclus dans cette évaluation.

\section{Cadres à l'échelle macro}

Les cadres à l'échelle macro décrivent le rôle de la collaboration dans la stimulation d'un changement social positif, ainsi que les facteurs essentiels à l'évaluation des initiatives de changement à grande échelle. Ils peuvent contribuer à saisir et à décrire les objectifs généraux du projet plurisectoriel de l'ASPC, ces derniers pouvant ensuite être reliés à certaines caractéristiques précises 
TABLEAU 3

Cadres aux échelles macro, méso et micro

\begin{tabular}{|c|c|c|}
\hline $\begin{array}{l}\text { Cadres à } \\
\text { l'échelle macro }\end{array}$ & Cadres à l'échelle méso & Cadres à l'échelle micro \\
\hline Innovation sociale & Cadre de changement des systèmes. & $\begin{array}{l}\text { Répertoire propositionnel pour la } \\
\text { conception et la mise en œuvre de } \\
\text { collaborations intersectorielles (en } \\
\text { leadership). }\end{array}$ \\
\hline Valeur commune & $\begin{array}{l}\text { Cadre orientant l'élaboration de stratégies } \\
\text { pour les organismes sans but lucratif. }\end{array}$ & $\begin{array}{l}\text { Cadre et processus pour des mesures en } \\
\text { santé publique fondées sur la } \\
\text { collaboration. }\end{array}$ \\
\hline \multirow[t]{7}{*}{ Impact collectif } & $\begin{array}{l}\text { Modèle permettant d'analyser la formation } \\
\text { dans les activités intersectorielles. }\end{array}$ & $\begin{array}{l}\text { Cadre RE-AIM pour évaluer l'impact des } \\
\text { partenariats plurisectoriels. }\end{array}$ \\
\hline & $\begin{array}{l}\text { Répertoire propositionnel pour la } \\
\text { conception et la mise en ouvre de } \\
\text { collaborations intersectorielles. }\end{array}$ & $\begin{array}{l}\text { Cadre de collaboration, d'évaluation et } \\
\text { d'amélioration. }\end{array}$ \\
\hline & $\begin{array}{l}\text { Cadre intégré pour une gouvernance } \\
\text { collaborative. }\end{array}$ & $\begin{array}{l}\text { Cadre pour comprendre l'incidence en } \\
\text { matière de rendement des réseaux } \\
\text { interorganisationnels. }\end{array}$ \\
\hline & $\begin{array}{l}\text { Cadre pour les résultats organisationnels de } \\
\text { la collaboration communautaire. }\end{array}$ & $\begin{array}{l}\text { Cadre pour évaluer l'efficacité des } \\
\text { réseaux de promotion de la santé. }\end{array}$ \\
\hline & $\begin{array}{l}\text { Cadre des antécédents, des processus et du } \\
\text { rendement perçu des collaborations } \\
\text { interorganisationnelles pour la prestation } \\
\text { de services publics. }\end{array}$ & $\begin{array}{l}\text { Indicateurs de rendement multiniveaux } \\
\text { pour les réseaux plurisectoriels et leur } \\
\text { gestion. }\end{array}$ \\
\hline & $\begin{array}{l}\text { Cadre collaboratif de création de valeur } \\
\text { pour analyser les partenariats d'affaires et } \\
\text { sans but lucratif. }\end{array}$ & \\
\hline & $\begin{array}{l}\text { Cadres et initiatives clés pour les } \\
\text { partenariats en soins de santé et en services } \\
\text { sociaux. }\end{array}$ & \\
\hline
\end{tabular}

des projets de partenariat financés. Les cadres à l'échelle macro comprennent les innovations sociales $^{33,34}$, la valeur partagée $^{35-37}$ et l'impact collectif ${ }^{38-42}$ (même si l'impact collectif a été regroupé avec d'autres cadres au niveau macro, nous sommes conscients qu'il présente certaines caractéristiques des cadres méso et micro). Discuter plus largement de chacun de ces cadres outrepasserait la portée de cet article. Notre synthèse présente néanmoins les points de vue de chacun d'entre eux, précise la façon dont sont envisagées les activités plurisectorielles et leur apport potentiel aux stratégies d'apprentissage et d'amélioration pour les partenariats plurisectoriels.

L'innovation sociale est décrite comme la recherche d'une « nouvelle solution à un problème social, plus efficace et plus durable que les solutions actuelles et dont la valeur profite avant tout à la société dans l'ensemble plutôt qu'à des intervenants du secteur privé » [traduction] ${ }^{43}$. Le Ber et Branzei $^{34}$ ont souligné que les partenariats étaient un élément clé de l'innovation sociale et qu'ils révélaient l'importance des liens entre les partenaires ainsi que leur confiance et leurs désillusions. Entretenir des relations plurisectorielles exige des partenaires de réévaluer en permanence leurs rôles et leurs liens en fonction de l'évolution des contextes et des circonstances.

À l'inverse, la valeur partagée favorise les investissements dans la compétitivité à long terme tout en faisant la promotion des objectifs sociaux et environnementaux ${ }^{35}$. Dans le cas où des partenariats sont établis en vue de générer une valeur partagée, les partenariats plurisectoriels offrent des outils incontournables pour l'obtention de résultats aussi bien opérationnels (augmentation du revenu, part de marché, rentabilité, etc.) réduction de l'empreinte carbone, compétences accrues, etc. $)^{37}$.

À la différence de la valeur partagée et de l'innovation sociale, avec l'impact collectif, l'accent est mis sur les partenariats que sociaux (meilleurs soins aux patients, plurisectoriels, qui sont influencés par cinq conditions principales : un programme commun, des systèmes de mesure partagés, des activités de renforcement, une communication constante et le soutien d'une organisation clé ${ }^{39-41}$. Un élément sous-jacent au succès des initiatives d'impact collectif est le processus d'apprentissage continu fondé sur des mesures partagées et des évaluations continues, qui permettent d'alimenter les indicateurs de processus et de résultats en lien avec l'évolution du partenariat $^{41}$.

\section{Cadres à l'échelle méso}

Dans notre analyse, les cadres à l'échelle méso sont ceux qui portent sur le fonctionnement des partenariats. Ceux qui sont présentés dans le tableau 3 se différencient par leur visée (par exemple la formation de partenariats), leurs facteurs de réussite et les résultats ou impacts prévus. Les thèmes les plus courants abordés dans ces cadres sont l'importance du contexte, le besoin de cerner clairement le problème, les processus nécessaires à l'établissement et au maintien de l'engagement des partenaires, l'importance de la compréhension et de l'interaction et enfin les liens avec les résultats du partenariat.

\section{Contexte}

Le contexte peut être considéré d'un point de vue extérieur comme interne. Le contexte extérieur comprend les normes, les ressources, les règlements et les activités des sociétés ainsi que les stratégies, les politiques et les conditions juridiques qui touchent le partenariat ${ }^{12,44,45}$.

Le contexte interne comprend les cultures, structures et politiques ministérielles et organisationnelles en place sur lesquelles les membres d'un organisme peuvent avoir une influence ${ }^{12}$, ainsi que la façon dont les caractéristiques et les interactions antérieures de chacun des organismes influence les partenariats et leurs résultats ${ }^{46}$.

\section{Cerner le problème}

De nombreux cadres à l'échelle méso révèlent l'importance de comprendre l'enjeu ou le problème abordé par le partenariat plurisectoriel, de même que les limites entre lesquelles il navigue (c'est-à-dire le contenu 
d'un système donné, par exemple les organismes, les relations, les histoires et les cultures) ${ }^{44}$. Les partenariats fructueux ont recours à différents groupes d'intervenants dans le cadre d'un processus explicite qui vise à intégrer divers points de vue. Il peut notamment s'agir d'élaborer des énoncés et des mandats, d'affecter des ressources et d'approuver des structures décisionnelles ${ }^{13,44}$.

\section{Processus de partenariat}

Les processus de partenariat regroupent les activités ordinaires des travaux relatifs au partenariat, comme l'établissement d'ententes, le renforcement du leadership et de la légitimité, la génération de la confiance entre les partenaires, la gestion des conflits et la planification des activités courantes liées au partenariat ${ }^{13}$. Le processus collaboratif de création de valeur va de pair avec le processus de partenariat et ses étapes d'évolution : au fil de la transformation des structures collaboratives, les structures relationnelles semblent se modifier pour améliorer le partage des ressources, intensifier les interactions, accroître les valeurs stratégiques et assurer une plus grande mobilisation envers les occasions d'innovation ${ }^{10,11}$. Il peut s'agir plus précisément d'offre de séances de formation, de rassemblement des ressources ou de contrôle des activités de mise en oeuvre $^{45}$. Les différentes étapes d'évolution des partenariats (établissement, sélection, mise en œuvre, conception et opérations, institutionnalisation) comportent de nombreux sous-processus : mécanismes visant à schématiser l'adaptation organisationnelle, à mettre en œuvre des processus officiels comme informels de gestion du risque ou encore à examiner différentes structures et conceptions favorisant l'expérimentation, dans le but d'obtenir une valeur partagée (comme le rassemblement d'équipes pour prendre des décisions communes, bâtir des relations de confiance et expérimenter l'autonomie organisationnelle) ${ }^{47}$.

\section{Interactions}

On recommande, pour de nombreux cadres, d'examiner les interactions entre les diverses composantes des partenariats afin de comprendre et d'améliorer ces derniers. C'est réalisable en se penchant sur l'harmonisation interorganisationnelle, les forces et faiblesses relatives des divers organismes (concurrents et collaborateurs), les obstacles qui les séparent et les déséquilibres de pouvoirs ${ }^{12}$. Le Cadre de changement des systèmes définit divers processus pour examiner les interactions entre les éléments du système, en particulier la façon dont on peut utiliser celles-ci pour provoquer un changement ${ }^{44}$. Introduire des processus capables de fournir une vue d'ensemble des interactions entre les organismes est sans doute une étape importante dans la compréhension du fonctionnement des relations plurisectorielles dans des contextes sociaux plus élargis.

\section{Résultats des partenariats}

Les résultats des partenariats peuvent être de premier degré (p. ex. résultats à court terme des travaux de partenariat), de deuxième degré (p. ex. mesures coordonnées, changements de pratique ou de perspective) ou de troisième degré ( $p$. ex. coévolution, formation de nouvelles institutions, nouvelles normes) ${ }^{13}$. Il peut également s'agir de changements (intentionnels ou non) d'états souhaités, de l'élaboration de nouveaux biens sociaux ou d'innovations technologiques, d'un meilleur apprentissage interorganisationnel, de l'amélioration des interactions entre les membres des divers organismes, d'un accès accru aux ressources, d'une plus grande capacité de servir les clients (si la prestation de service est une activité) et enfin d'une meilleure capacité de résolution des problèmes ${ }^{48}$. Étant donné cette diversité, il est essentiel que les mesures de résultats soient adaptées à chaque partenariat et à son stade d'évolution.

\section{Cadres à l'échelle micro}

Les cadres à l'échelle micro définissent les piliers sur lesquels s'appuie le fonctionnement des partenariats ainsi que les indicateurs des activités de partenariat (en particulier des outils spécifiques de collecte de données).

Parmi les cadres à l'échelle micro que nous avons analysés, trois portent sur des réseaux interorganisationnels ${ }^{49-51}$, les autres relevant plutôt de collaborations et de partenariats. En partant de ce point de vue, le cadre et les processus pour la collaboration en santé publique ont défini cinq piliers des partenariats : évaluation et planification de la collaboration, mise en œuvre de mesures ciblées, modification des conditions dans les communautés et les systèmes, changement dans les comportements et enfin amélioration de la santé et de l'équité en santé ${ }^{52}$. On a attribué à ces piliers des indicateurs précis de succès, comme la présence d'un but commun, des modèles logiques clairs, des rôles explicites ainsi qu'un leadership reconnu et bien réparti.

En dépit de difficultés persistantes à établir des liens de cause à effet entre la coordination et le rendement d'un réseau, Gulati et collab. ${ }^{49}$ ont proposé trois piliers expliquant la réussite d'un réseau (la portée, la richesse et la réceptivité) et ils ont fourni des indicateurs précis pour chacun d'eux, comme la distance entre les partenaires, la confiance, l'engagement et la multiplexité du lien. Le Health Promotion Networks Framework $^{50}$ met plutôt l'accent sur la structure, le processus et l'efficacité et présente divers indicateurs comme l'âge, la taille et la forme du réseau, les processus facilités par le réseau (comme la défense des intérêts, la formation et la sensibilisation du public), les effets de l'apprentissage organisationnel et les changements de pratique. De son côté, le Collaborative Evaluation Improvement Framework $^{53}$ fournit des stratégies de collecte de données précises afin d'assurer l'efficacité du réseau: vision d'ensemble ou schémas de l'équipe et des procédures décisionnelles, réalisation d'entrevues, de sondages et d'analyses de documents afin de mieux comprendre les processus internes et enfin collecte de données sur la qualité des interactions de l'équipe à l'aide d'outils précis (comme le Levels of Organizational Integration Rubric $[\mathrm{LOIR}]^{53}$ et le Team Collaboration Assessment Rubric [TCAR ${ }^{53}$ ).

\section{Stratégie d'apprentissage et d'amélioration}

La figure 1 présente la stratégie d'apprentissage et d'amélioration en six volets du CPCMC pour l'initiative des partenariats plurisectoriels. Ces cycles d'apprentissage, fondés sur les étapes d'apprentissage de Kolb (sensation, observation, réflexion et action) ${ }^{31}$, permettent au CPCMC de poser rapidement les questions de fond qui s'imposent à propos des partenariats plurisectoriels, de recueillir et analyser l'information nécessaire en se fondant sur des 
FIGURE 1

Composantes de la stratégie d'apprentissage et d'amélioration

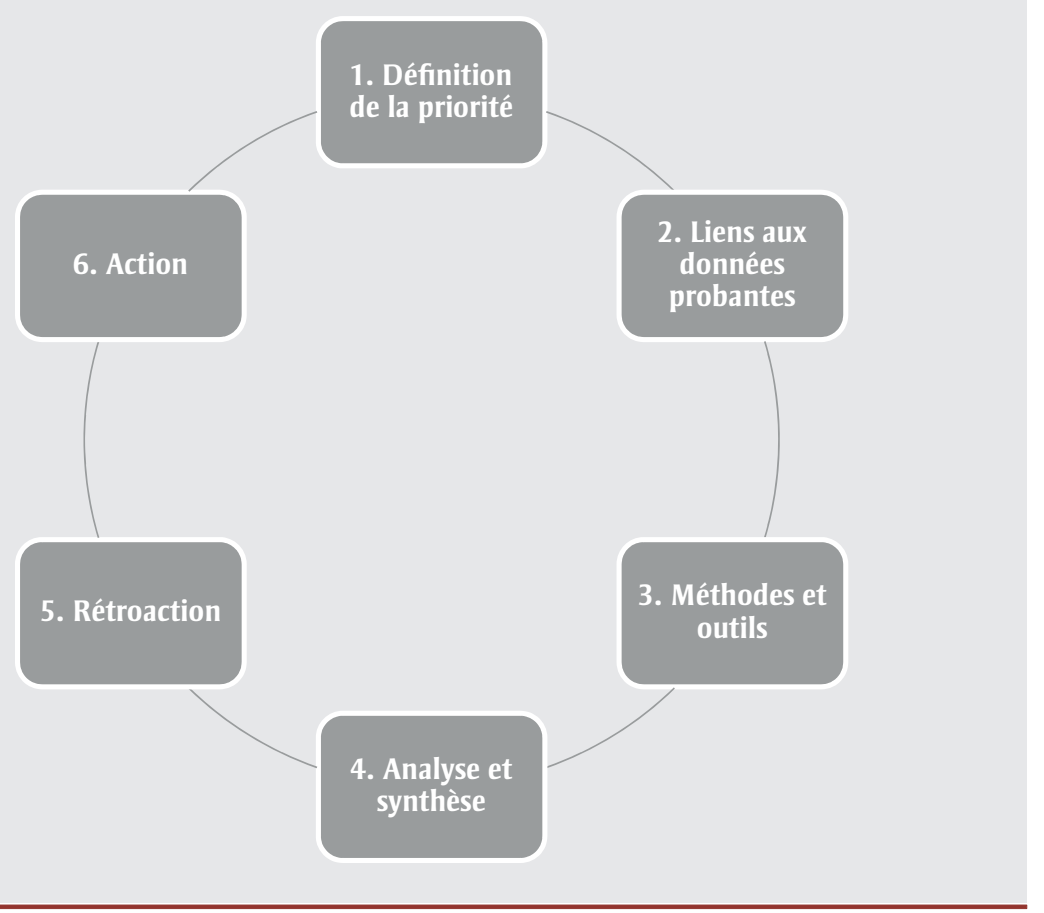

données probantes et de la présenter de manière accessible au personnel du CPCMC (voire à d'autres personnes).

\section{1. Établissement des priorités - préciser les besoins en matière d'apprentissage et d'amélioration relativement aux parte- nariats plurisectoriels et déterminer leur niveau de priorité}

Les consultations initiales réalisées auprès $\mathrm{du}$ personnel du CPCMC ont fourni un aperçu du projet plurisectoriel du CPCMC, ce qui a permis de définir diverses orientations potentielles pour l'apprentissage et l'amélioration (figure 2). À partir des hypothèses orientant le projet plurisectoriel à son point de départ (élargissement de la portée, utilisation des ressources et accroissement de l'impact), ces besoins en matière d'apprentissage ont permis de formuler des questions précises tout au long de la stratégie d'apprentissage et d'amélioration. L'élaboration de ces questions prioritaires requiert la participation des membres et des équipes du CPCMC, voire des organismes ayant conclu des partenariats avec le CPCMC dans le cadre des projets de partenariats plurisectoriels. Ces processus d'établissement des priorités se font lors d'ateliers en personne ou par l'application de processus Delphi modifiés pour recueillir les points de vue d'un grand groupe ${ }^{54}$.

2. Lien aux données probantes - confronter les besoins prioritaires en matière d'apprentissage et la réalité des cadres de partenariat

Le volet 2 s'appuie sur la littérature pertinente pour déterminer comment combler les besoins en matière d'apprentissage du CPCMC et comment cerner et résoudre les problèmes. Il s'agit notamment de lier les domaines prioritaires aux cadres de partenariat pertinents à l'aide d'un certain nombre de critères d'harmonisation, notamment le niveau auquel on souhaite obtenir l'information, l'étape d'évolution à laquelle se situe le partenariat et le niveau de détail nécessaire. Par exemple, un des besoins clés du CPCMC en matière d'apprentissage consiste à saisir la portée des projets de partenariats plurisectoriels actuels. La schématisation, grâce aux cadres conceptuels du partenariat, aide à déterminer les différentes composantes de la portée (p. ex. cibler les personnes, les organismes, les secteurs, les communautés), les facteurs qui influencent cette portée et la façon dont ces connaissances peuvent contribuer à améliorer l'initiative de partenariats du CPCMC.

3. Méthodes et outils - recueillir de l'information en vue de combler les besoins en matière d'apprentissage liés aux partenariats plurisectoriels

Les cadres analysés ont permis de voir comment on pouvait définir un besoin en matière d'apprentissage lié à un engagement plurisectoriel, ainsi que les outils nécessaires au recueil des données pertinentes. À la base, il faut déterminer à quel niveau l'information est recherchée (initiative générale, projet, organisme) ainsi que le stade de développement du partenariat. La littérature sur les apprentissages organisationnels révèle que l'information au niveau de l'organisme porte sur ses rôles, ses structures et ses processus internes, sur sa culture d'apprentissage ainsi que sur les avantages organisationnels du partenariat. Par exemple, dans le cadre de la première priorité du CPCMC en matière d'apprentissage (cibler sa portée), les données pertinentes ont pu être recueillies dans divers documents, dont les rapports des projets, et dans les entrevues menées auprès d'informateurs clés (personnel du CPCMC et de l'ASPC et collaborateurs externes).

$\mathrm{Au}$ niveau du projet, il est possible de cibler la schématisation des relations interorganisationnelles au sein des projets de partenariat, la définition et la schématisation des communautés de pratique et le suivi des étapes de collaboration au sein des projets. Cela permet de comprendre comment les individus et les organismes collaborent dans le cadre de projets de partenariat, d'identifier les voies de communication disponibles et de mesurer la fréquence et l'intensité des communications pendant ces projets ${ }^{55}$. Afin de recueillir de l'information sur ces aspects clés, de nouvelles méthodes de collecte (comme l'analyse des réseaux sociaux) pourraient s'ajouter aux techniques qualitatives et quantitatives traditionnelles ${ }^{53}$.

$\mathrm{Au}$ niveau de l'initiative générale, le CPCMC aurait besoin de données sur l'ensemble des projets de partenariat pour mieux connaître les premières étapes de 
FIGURE 2

- Comprendre et diffuser les points de vue de l'ASPC et des organismes partenaires.

- Examiner les effets de la conception organisationnelle interne de l'ASPC (notamment ses principales fonctions d'entreprise) sur les activités plurisectorielles ainsi que la réussite des projets et voir comment elle pourrait être revue.

- Mesurer la crédibilité du concept de partenariats plurisectoriels ainsi que le rôle du gouvernement dans l'initiative.

- Montrer comment on tire parti des fonds du secteur privé et des contributions en nature pour créer de la valeur sociale et stimuler des changements de comportement.

- Voir comment cette méthode de travail offre des possibilités d'innovation sociale.

- Démontrer qu'une approche plurisectorielle a une incidence sur les déterminants comportementaux et environnementaux de la santé.

- Examiner les conséquences imprévues (positives et négatives) du programme.

- Voir comment établir des partenariats dans des situations ou circonstances difficiles, p. ex. en santé autochtone ou en milieu de travail.

- Définir les compétences nécessaires pour pouvoir trouver et établir des partenariats (Quelles sont ces compétences? Le personnel de l'ASPC les possède-t-il? Peuvent-elles être améliorées? Quelles formations pourraient être utiles?).

- Examiner les aspects du système de rémunération au rendement, notamment la pertinence des objectifs et des mesures.

- Déterminer comment les organismes partenaires peuvent partager de l'information au sein de leurs propres réseaux organisationnels.

l'établissement de ces partenariats, en particulier sur les conditions incontournables de leur impact collectif (et leurs indicateurs connexes), comme l'établissement d'un programme commun et de mesures partagées. Pour recueillir de l'information sur le projet plurisectoriel du CPCMC, seraient pertinents comme indicateurs la façon dont les partenaires et la communauté définissent et formulent le problème, le niveau de participation anticipé des partenaires au système partagé de mesures ainsi que les changements dans les activités des partenaires visant l'harmonisation avec le plan d'action partagé $^{41}$. Pour les partenariats en place depuis longtemps ou en voie d'achèvement, l'information au niveau de l'initiative générale pourrait porter davantage sur ses résultats, utilisant des techniques de schématisation ou la technique du « changement le plus significatif » (« most significal change ») afin d'en décrire les impacts ${ }^{56}$. Cette stratégie d'apprentissage et d'amélioration ouvre au CPCMC de vastes possibilités pour concevoir, mettre à l'essai et améliorer les indicateurs permettant de mesurer l'efficacité de ses partenariats.

\section{Analyse et synthèse - application aux données de perspectives analytiques pertinentes}

Le volet 4 de la stratégie permet d'appliquer des méthodes pertinentes et rigoureuses à l'analyse et à la synthèse de données provenant de divers contextes et recueillies au moyen de méthodologies variées. Par exemple, une synthèse réaliste aide à repérer ce qui fonctionne et à déterminer pourquoi et comment certaines activités ont tel effet dans tel contexte ${ }^{57}$. Dans le cas du CPCMC, l'application d'une perspective réaliste favoriserait le regroupement de données probantes hétérogènes et la génération d'une orientation stratégique lors des discussions de groupe (visant à dégager une interprétation commune à partir de la nouvelle information). Les avis recueillis sont susceptibles de mettre en lien les données et les personnes ou groupes pertinents afin de favoriser la compréhension, l'évaluation, la résolution de problèmes et l'application des pratiques de partenariat du CPCMC.

L'un des éléments essentiels du cycle d'apprentissage est de s'assurer que l'information est accessible au public 
auquel elle est destinée. Cependant, bien que la stratégie d'apprentissage et d'amélioration soit principalement destinée au CPCMC, les cycles d'apprentissage proposés permettront d'obtenir également les avis d'autres personnes et groupes (du gouvernement et à l'extérieur). Il peut notamment s'agir de rédiger des rapports techniques, diverses publications ou des cartes interactives en ligne, de faire des présentations aux divers membres de l'équipe du CPCMC ainsi qu'aux employés d'autres secteurs et ordres de gouvernement ou encore d'organiser des ateliers avec différents groupes selon l'interprétation et le traitement des données.

5. et 6. Rétroaction et action - refonte des processus, des structures, des stratégies d'évaluation et des options de mobilisation internes pour les partenariats plurisectoriels

Grâce aux séances de rétroaction tenues à la fin de chaque cycle d'apprentissage (volet 5), les membres et équipes du CPCMC peuvent adapter leurs nouveaux apprentissages aux changements organisationnels, stratégiques ou de pratiques envisagés. Ils peuvent notamment revoir les processus internes du CPCMC, modifier les techniques d'établissement de partenariats, élaborer de nouveaux modules de formation, mettre en œuvre des mécanismes de gouvernance des partenariats (aussi bien pour les nouveaux que pour ceux en cours) ou revoir les procédures d'évaluation des impacts et des résultats. Chaque action peut avoir une incidence sur les piliers prioritaires d'apprentissage (voir volet 1) et donc contribuer à déplacer la cible au cours du cycle d'apprentissage suivant. Avec ce degré de souplesse, la stratégie d'apprentissage et d'amélioration du CPCMC va rester pertinente et utile au fil des changements à venir au sein des projets de partenariats plurisectoriels.

\section{Analyse}

Les partenariats interorganisationnels constituent un élément important de l'initiative du Canada visant à résoudre des problèmes de santé publique complexes par la prévention des maladies chroniques, l'adoption d'un mode de vie plus sain et la réduction des inégalités en matière de santé $^{1,9}$. Ces partenariats cherchent à accroître la portée des programmes fondés sur des données probantes, à tirer parti de nouvelles ressources et à stimuler le changement dans la santé et la culture des communautés et des organismes partenaires. Insister sur l'engagement dans des partenariats est conforme aux intentions du gouvernement, comme l'illustre un récent Discours du Trône, de saisir « [...] les occasions présentées par la finance sociale et l'Appel national d'idées sur la finance sociale, qui s'est avéré une réussite ${ }^{58}$.

Le projet plurisectoriel du CPCMC, qui compte de nombreux partenaires traditionnels comme non traditionnels, vise à réaliser des gains sociaux et économiques en tirant parti de l'expertise, des ressources et de l'influence de ses divers partenaires. Dans cet article, on a décrit la méthode utilisée par le CPCMC pour élaborer une stratégie d'apprentissage et d'amélioration pour les partenariats plurisectoriels. Bien qu'elle soit principalement destinée au CPCMC, cette stratégie peut s'appliquer à d'autres groupes et organismes, gouvernementaux ou non.

La stratégie d'apprentissage et d'amélioration fondée sur des données probantes pour les partenariats plurisectoriels que nous avons décrite est conforme aux orientations actuelles de la recherche interventionnelle en santé de la population $^{59,60}$. L'initiative des partenariats plurisectoriels du CPCMC porte sur l'ensemble des interventions en santé de la population : l'action précède la science, les innovations sont mises en œuvre par une équipe responsable de la politique et de la pratique, un large éventail de connaissances pertinentes est utilisé pour définir et comprendre l'initiative, les efforts sont habités par le désir de stimuler un changement social à grande échelle et enfin il faut du temps pour que les résultats de l'initiative se manifestent ${ }^{61}$. Contrairement aux méthodes de recherche fondées sur une hypothèse de départ, ce type d'intervention dans le domaine de la santé de la population fait appel à un modèle de recherche intégré permettant de comprendre, d'évaluer et de diffuser de manière rigoureuse la manière dont fonctionnent les pratiques, dans quelles conditions, pour qui et pourquoi $^{61}$. C'est cette approche « apprenons en fonctionnant » qui est à la base de la stratégie d'apprentissage et d'amélioration du CPCMC pour les partenariats plurisectoriels, et qui se révèle très pertinente également pour d'autres initiatives gouvernementales (de l'ASPC et d'autres ministères). Les priorités d'apprentissage de départ (compréhension de la portée du partenariat, effets attendus et imprévus et repérage des connaissances fondées sur les pratiques) ont grandement contribué à la littérature scientifique sur les partenariats et à soutenir les efforts du CPCMC - puis ceux d'autres ministères - dans l'amélioration des partenariats plurisectoriels.

\section{Points forts et limites}

Cette étude a deux limites principales. D'abord, comme les consultations n'ont été menées qu'auprès du personnel du CPCMC, il n'a pas été possible d'obtenir les points de vue et les expériences d'autres personnes participant aux projets de partenariats plurisectoriels. À mesure que la stratégie d'apprentissage et d'amélioration pour les partenariats plurisectoriels sera mise en œuvre, il sera important d'élargir l'éventail des participants à d'autres directions et divisions de l'ASPC et aux organismes partenaires. Il est prévu d'obtenir ces points de vue sur la portée par la collecte de données auprès des partenaires externes (des secteurs public et privé).

En second lieu, même si l'analyse de la littérature sur les cadres a été systématique, elle pourrait ne pas être exhaustive : d'autres cadres et modèles pertinents n'ont peut-être pas été inclus dans l'étude. Néanmoins, les cadres évalués ont fourni des points de vue variés sur les structures, les fonctions et les résultats des partenariats plurisectoriels.

Un premier cycle de la stratégie visera la compréhension de la portée des projets de partenariat actuels, un second cycle les conséquences imprévues (positives ou non) des différents projets de partenariat. Les conclusions tirées à la suite de ces deux cycles s'ajouteront aux efforts d'augmentation des connaissances sur les expériences pratiques des participants dans leurs projets de partenariat. 


\section{Conclusion}

Dans cet article, nous avons retracé dans ses grandes lignes la méthode utilisée par le CPCMC pour augmenter et améliorer les connaissances sur les partenariats plurisectoriels. La stratégie décrite ici fournit au CPCMC - et peut-être également à d'autres parties - des moyens pratiques, flexibles et fondés sur des données probantes de cerner et de combler les principaux besoins en matière d'apprentissage liés aux partenariats plurisectoriels, tout en permettant de répondre aux demandes des acteurs souhaitant modifier les politiques publiques. Quoique l'établissement de tels partenariats pour traiter des enjeux de santé complexes ne soit pas nouveau, nos connaissances à leur sujet sont limitées. La stratégie d'apprentissage et d'amélioration pour ces partenariats dont cet article a fait état aidera, souhaitons-le, le CPCMC et d'autres organismes à combler les lacunes en matière de connaissances et à accroitre la capacité des projets plurisectoriels à s'attaquer aux questions de santé qui affectent les Canadiens.

\section{Références}

1. Johnston LM, Finegood DT. Cross-Sector partnerships and public health: challenges and opportunities for addressing obesity and noncommunicable diseases through engagement with the private sector. Annu Rev Public Health. 2015;36:255-71. doi: 10.1146/annurev-publhealth-031914-122802

2. Woulfe J, Oliver TR, Zahner SJ, Siemering KQ. Multisector partnerships in population health improvement. Prev Chronic Dis. 2010;7(6):A119.

3. Reich MR. Public-private partnerships for public health. Cambridge (MA) : Harvard Centre for Population Development Studies; 2002.

4. Provan KG, Fish A, Sydow J. Interorganizational networks at the network level: a review of the empirical literature on whole networks. J Manage. 2007;33(3):479516. doi: $10.1177 / 0149206307302554$

5. Provan KG, Beagles JE, Leischow SJ. Network formation, governance, and evolution in public health: the North American Quitline Consortium case. Health Care Manage Rev. 2011;36 (4):315-326. doi: 10.1097/HMR.0b013e31820 e1124
6. Provan KG, Lemaire R. Core concepts and key ideas for understanding public sector organizational networks: using research to inform scholarship and practice. Public Adm Rev. 2012;72(5):638-48. doi: 10.1111/ j.1540-6210.2012.02595.x

7. Willis CD, Riley BL, Best A, Ongolo-Zogo P. Strengthening health systems through networks: the need for measurement and feedback. Health Policy Plan. 2012;27Suppl 4:62-6.

8. Willis CD, Riley BL, Herbert CP, Best A. Networks to strengthen health systems for chronic disease prevention [Internet]. Am J Public Health. 2013;103(11):e39-e48. doi: 10.2105/AJPH.2013.301249

9. Agence de la santé publique du Canada. Partenariats plurisectoriels pour promouvoir les modes de vie sains et prévenir les maladies chroniques [en ligne]. Ottawa (Ont.) : Agence de la santé publique du Canada; 2014 [consulté le 21 avril 2015]. En ligne à: http://www.phac-aspc.gc.ca/fo-fc/ mspphl-pppmvs-fra.php

10. Austin JE, Seitanidi MM. Collaborative value creation: a review of partnering between nonprofits and businesses. Part 2 . Partnership processes and outcomes. Nonprof Volunt Sec Q.2012;41(6):929-968.

11. Austin JE, Seitanidi MM. Collaborative value creation: a review of partnering between nonprofits and businesses. Part 1 . Value creation spectrum and collaboration stages. Nonpro Volunt Sec Q.2012;41(5): 726-758.

12. Al-Tabbaa O, Leach D, March J. Collaboration between nonprofit and business sectors: a framework to guide strategy development for nonprofit organizations. Voluntas: Int J Volunt Nonprof Org. 2013; 25(3):657-678. doi: 10.1007/s11266-0139357-6

13. Bryson JM, Crosby BC, Stone MM. The design and implementation of cross-sector collaborations: propositions from the literature. Public Admin Rev. 2006;66(Suppl. 1)44-55.

14. Friedman SR, Reynolds J, Quan MA, Call S, Crusto CA, Kaufman JS. Measuring changes in interagency collaboration: an examination of the Bridgeport Safe Start Initiative. Eval Program Plann. 2007;30 (3):294-306
15. Kothari A, Sibbald SL, Wathen CN. Evaluation of partnerships in a transnational family violence prevention network using an integrated knowledge translation and exchange model: a mixed methods study. Health Res Policy Syst. 2014;12(1):25. doi: $10.1186 / 1478-4505-12-25$

16. Kumaresan J, Heitkamp P, Smith I, Billo N. Global Partnership to Stop TB: a model of an effective public health partnership. Int $\mathrm{J}$ Tuberc Lung Dis. 2004;8(1):120-129.

17. Kernaghan K. Partnership and public administration: conceptual and practical considerations. Can Public Admin. 1993; 36(1):57-76.

18. Butterfoss FD. Coalitions and partnerships in community health. San Francisco (CA) : Jossey-Bass; 2007.

19. Asante AD, Zwi AB. Public-private partnerships and global health equity: prospects and challlenges. Indian J Med Ethics. 2007; 4(4):176-180.

20. Barr DA. Ethics in public health research: a research protocol to evaluate the effectiveness of public-private partnerships as a means to improve health and welfare systems worldwide. Am J Public Health. 2007;97(1):19-25.

21. Bate SP, Robert G. Knowledge management and communities of practice in the private sector: Lessons for modernizing the NHS in England and Wales. Public Administration. 2002;80:643-663. doi: 10.1111/14679299.00322

22. Beaglehole R, Ebrahim S, Reddy S, Voute J, Leeder S. Prevention of chronic diseases: a call to action. Lancet. 2007;370(9605):2152-2157.

23. Galea G, McKee M. Public-private partnerships with large corporations: setting the ground rules for better health. Health Policy. 2014;115(2-3):138-140. doi: 10.1016/j. healthpol.2014.02.003

24. Leenaars K, Jacobs-van der Bruggen $M$, Renders C. Determinants of successful public-private partnerships in the context of overweight prevention in Dutch youth. Prev Chronic Dis. 2013;10:E117 doi: 10.5888/ pcd10.120317

25. Mannar MG, van Ameringen M. Role of public-private partnership in micronutrient food fortification. Food Nutr Bull. 2003;24(4 Suppl):S151-4. 
26. Rummery K. Healthy partnerships, healthy citizens? An international review of partnerships in health and social care and patient/user outcomes. Soc Sci Med. 2009;69 (12):1797-1804. doi: 10.1016/j.socscimed. 2009.09.004

27. Weiner BJ, Alexander JA. The challenges of governing public-private community health partnerships. Health Care Manage Rev. 1998;23(2):39-55

28. Widdus R. Public-private partnerships: an overview. Trans $\mathrm{R}$ Soc Trop Med Hyg. 2005;99Suppl 1:S1-8.

29. Senge PM. The fifth discipline: the art and practice of the learning organization. New York : Currency; 1994.

30. Senge PM, Sterman JD. Systems thinking and organizational learning: acting locally and thinking globally in the organization of the future. Eur J Oper Res. 1992;59:137-150.

31. Kolb DA. Experiential learning: experience as the source of learning and development. Englewood Cliffs (NJ) : Prentice Hall; 1984.

32. Deming WE. Out of the crisis. Cambridge (MA) : MIT Press; 2000.

33. Cajaiba-Santana G. Social innovation: moving the field forward. A conceptual framework. Technol Forecast Soc.2014;82 (0):42-51 .

34. Le Ber MJ, Branzei O. (Re)forming strategic cross-sector partnerships: Relational processes of social innovation. Bus Soc. 2010;49 (1):140-172. doi:10.1177/0007650309345457

35. Porter M, Kramer M. Creating shared value. Harvard Bus Rev. 2011 Jan/Feb.

36. Porter ME, Hills G, Pfitzer M, Patscheke S, Hawkins E. Measuring shared value. How to unlock value by linking social and business results. Boston (MA) : FSG; 2011.

37. Bockstette V, Stamp M. Creating shared value: a how-to guide for the new corporate (r)evolution. Boston (MA) : FSG; 2011.

38. Hanleybrown F, Kania J, Kramer M. Channeling change: making collective impact work [en ligne]. Stanford Soc Innov Rev. 2012 [consulté le 21 avril 2015]. En ligne à : http://ssir.org/articles/entry/channeling_ change_making_collective_impact_work
39. Kania J, Kramer M. Embracing emergence: how collective impact addresses complexity. Stanford Soc Innov Rev [en ligne]. 2013 [consulté le 21 avril 2015]. En ligne à : http:// www.ssireview.org/blog/entry/embracing_ emergence_how_collective_impact_addresses_ complexity

40. Kania J, Kramer M. Collective impact [en ligne]. Stanford Soc Innov Rev. 2011 [consulté le 21 avril 2015]. En ligne à : http://www. ssireview.org/articles/entry/collective_impact

41. Preskill H, Parkhurst M, Splansky Juster J. Guide to evaluating collecting impact: assessing progress and impact. Boston (MA) : Collective Impact Forum; 2013.

42. Preskill H, Parkhurst M, Splansky Juster J. Guide to evaluating collecting impact: Learning and evaluation in the collective impact context. Boston (MA) : Collective Impact Forum; 2013.

43. Phills JA, Deiglmeier K, Miller DT. Rediscovering social innovation. Stanford Soc Innov Rev. 2008;6(4):34-43.

44. Foster-Fishman PG, Nowell B, Yang H. Putting the system back into systems change: a framework for understanding and changing organizational and community systems. Am J Community Psychol. 2007;39(3-4):197-215.

45. Emerson K, Nabatchi T, Balogh S. An Integrative Framework for Collaborative Governance. J Public Admin Res Theor. 2011;22 (1):1-29.

46. Seitanidi MM, Koufopoulos DN, Palmer P. Partnership Formation for Change: Indicators for Transformative Potential in Cross Sector Social Partnerships. Journal of Business Ethics. 2010;94(S1):139-161.

47. Chen B. Antecedents or processes? Determinants of perceived effectiveness of interorganizational collaborations for public service delivery. Int Public Manag J. 2010;13 (4):381-407.

48. Nowell B, Foster-Fishman P. Examining mMulti-Sector Community Collaboratives as Vehicles for Building Organizational Capacity. Am J Community Psychol. 2011;48(34):193-207. doi: 10.1007/s10464-010-9364-3

49. Gulati R, Lavie D, Madhavan R. How do networks matter? The performance effects of interorganizational networks. Res Organ Behav. 2011;31:207-224.
50. Dietscher C. How can the functioning and effectiveness of networks in the settings approach of health promotion be understood, achieved and researched? Health Promot Int. 2013 doi: 10.1093/heapro/dat067.

51. Herranz J. Multilevel performance indicators for multisectoral networks and management. Am Rev Public Admin. 2010;40 (4):445-60. doi:10.1177/0275074009341662

52. Fawcett S, Schultz J, Watson-Thompson J, Fox M, Bremby R. Building multi-sectoral partnerships for population health and health equity. Prev Chronic Dis. 2010;7(6).

53. Woodland RH, Hutton MS. Evaluating organizational collaborations: Suggested entry points and strategies. Am J Eval. 2012;33 (3):366-83. doi: 10.1177/1098214012440028

54. Hsu CC, Sandford BA. The Delphi Technique: making sense of consensus. Pract Assess Res Eval. 2007;12(10):1-8.

55. Glasby J, Dickinson H, Miller R. Partnership working in England-where we are now and where we've come from. Int $\mathrm{J}$ Integr Care. 201111 Spec Ed

56. McIsaac E, Van Ymeren J. Demonstrating the results of capacity building: Final report of the Partnerships Grant Learning Project. Toronto (Ont.). The Mowat NFP; 2014.

57. Pawson R. The science of evaluation: a realist manifesto. London (UK) : Sage Publications; 2013.

58. Gouverneur général Canada. Discours du Trône ouvrant la deuxième session, quarante unième législature du Canada. 16 octobre 2013; [consulté le 21 avril 2015]. En ligne à : http://www.lop.parl.gc.ca/ParlInfo/Documents/ThroneSpeech/41-2-f.html

59. Hawe P, Potvin L. What is population health intervention research? Can J Public Health. 2009;100(1). Suppl I8-14

60. Rychetnik L, Frommer M, Hawe P, Shiell A. Criteria for evaluating evidence on public health interventions. J Epidemiol Community Health. 2002;56(2):119-127.

61. Hobin E, Hayward S, Riley BL, Di Ruggiero E, Birdsell J. Maximising the use of evidence: exploring the intersection between population health intervention research and knowledge translation from a Canadian perspective. Evid Pol. 2012;8(1):97-115. 